\title{
Laparoscopic Resection of Isolated Retroperitoneal Gastric Duplication Cyst in an Infant
}

\author{
Vipul Gupta, Usman Javaid, Ghadir Jaber, Diary Mohd and Mamoun AIMarzouqi \\ Department of Pediatric Surgery and Urology, Latifa Women and Children Hospital, Dubai, United Arab Emirates
}

\begin{abstract}
We, herein report a rare case of retroperitoneal gastric duplication cyst in a 5-month female infant presenting with antenatal diagnosis of right renal cyst. Post-natal evaluation showed presence of retroperitoneal cyst in the region of right adrenal gland, which progressively increased in size on serial imaging. Laparoscopic resection resulted in satisfactory recovery. Intra-operative findings and histopathology confirmed diagnosis of isolated retroperitoneal gastric duplication cyst. Authors present the clinical features of this rare entity along with feasibility of successful laparoscopic excision in infantile age group.
\end{abstract}

Key Words: Gastric duplication cyst, Infants, Laparoscopy.

How to cite this article: Gupta V, Javaid U, Jaber G, Mohd D, AIMarzouqi M. Laparoscopic resection of isolated retroperitoneal gastric duplication cyst in an infant. J Coll Physicians Surg Pak. 2019; 29 (Supplement 2):S141-S143.

\section{INTRODUCTION}

Gastric duplication cyst (GDC) remains relatively rare congenital malformation accounting for about $9 \%$ of all gastrointestinal duplications.1-3 Among the reported cases of GDC, isolated retroperitoneal cystic gastric duplication remains extremely uncommon in pediatric age group with most of the cases presenting in childhood. ${ }^{4}$ A review of available English literature suggests only a few cases being reported in infancy and neonatal period till date.1-4 The present case warrants reporting, owing to clinical presentation mimicking adrenal cyst in infant and its successful management with minimally invasive surgery. The clinical features along with pertinent literature are reviewed and surgical innovation in planning intra-abdominal access site, which facilitated complete excision even in presence of unusual retroperitoneal location of GDC is highlighted.

\section{CASE REPORT}

A 5-month full term female baby was evaluated in postnatal period for right antenatal hydronephrosis. Postnatal ultrasound scan at day 7 of life showed presence of $3 \mathrm{~cm} \times 2.5 \mathrm{~cm}$ lobulated cystic lesion in the region of right suprarenal gland, suggestive of adrenal hemorrhage. Clinical examination showed soft nondistended abdomen with no palpable intra-abdominal mass. Laboratory investigations showed normal renal

Correspondence to: Dr Vipul Gupta, Department of Pediatric Surgery and Urology, Latifa Women and Children Hospital,

Dubai, United Arab Emirates

E-mail: drvipul7577@gmail.com

Received: April 04, 2019; Revised: July 15, 2019;

Accepted: July 27, 2019 functions. Patient was reviewed in clinic after 4 months with repeat ultrasound scan abdomen, which showed progressive increase in the size of anechoic cystic lesion measuring $4 \mathrm{~cm} \times 3 \mathrm{~cm}$ in right suprarenal region with few internal echoes, suggestive of adrenal hemorrhage, with normal non-hydronephrotic both kidneys and intraabdominal solid visceras (Figure 1). Renal scintigraphy showed both kidneys normal with split renal function of $47 \%$ in right and $53 \%$ in left kidney with presence of right suprarenal lesion compressing the right kidney. Due to progressive increase in size of cyst to $5 \mathrm{~cm}$ on repeat ultrasound scan at 6 months of age, further evaluation was done with magnetic resonance imaging (MRI). MRI scan showed $7 \mathrm{~cm} \times 4 \mathrm{~cm} \times 6 \mathrm{~cm}$ heterogeneous lesion in right suprarenal region, compressing the right kidney without any evidence of infiltration in surrounding structures (Figure 2). The lesion appeared to have thin wall on MRI which enhanced on contrast administration. Clinical examination showed presence of palpable soft non-tender cystic mass in right upper abdomen. Laboratory investigations showed normal blood count, renal and liver function tests. With possibility of cystic neuroblastoma versus adrenal cyst, the patient underwent laparoscopy, which was performed with patient in semi-left side lying position. The margins of mass were delineated under anesthesia and optical port was inserted $3 \mathrm{~cm}$ to the right of umbilicus with open technique to provide better visualisation and access for dissection (Figure $3 \mathrm{a}-\mathrm{c}$ ). Two $5 \mathrm{~mm}$ ports were inserted in right mid-axillary and mid-clavicular line, by following the principle of triangulation. One $3 \mathrm{~mm}$ port was inserted in epigastric region for liver retractor. Intraoperative findings showed presence of cystic mass in retroperitoneal region separated from right kidney and surrounding structures (Figure $3 \mathrm{~d}$ ). The mass was 
carefully dissected from surrounding structures and retrieved through endo-bag after aspiration of around $175 \mathrm{ml}$ of straw coloured fluid. There was neither sharing of common wall nor communication with any part of gastrointestinal tract (GIT). The patient recovered well in postoperative period and was dis-charged on second postoperative day. Histopathology showed presence of gastric smooth muscle with mucosal lining in cyst wall, thus confirming diagnosis of isolated GDC. On 7 months follow-up, patient remained asymptomatic with no evidence of any intra-abdominal mass on ultrasound scan abdomen repeated twice.

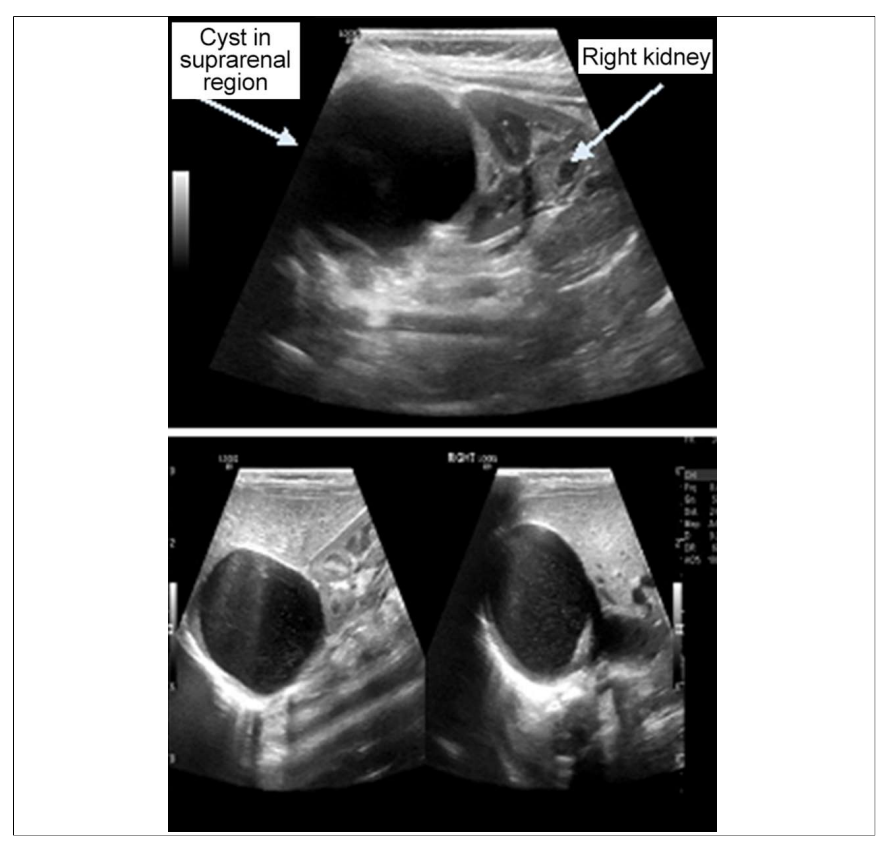

Figure 1: Ultrasound scan showing cystic lesion in right suprarenal region.

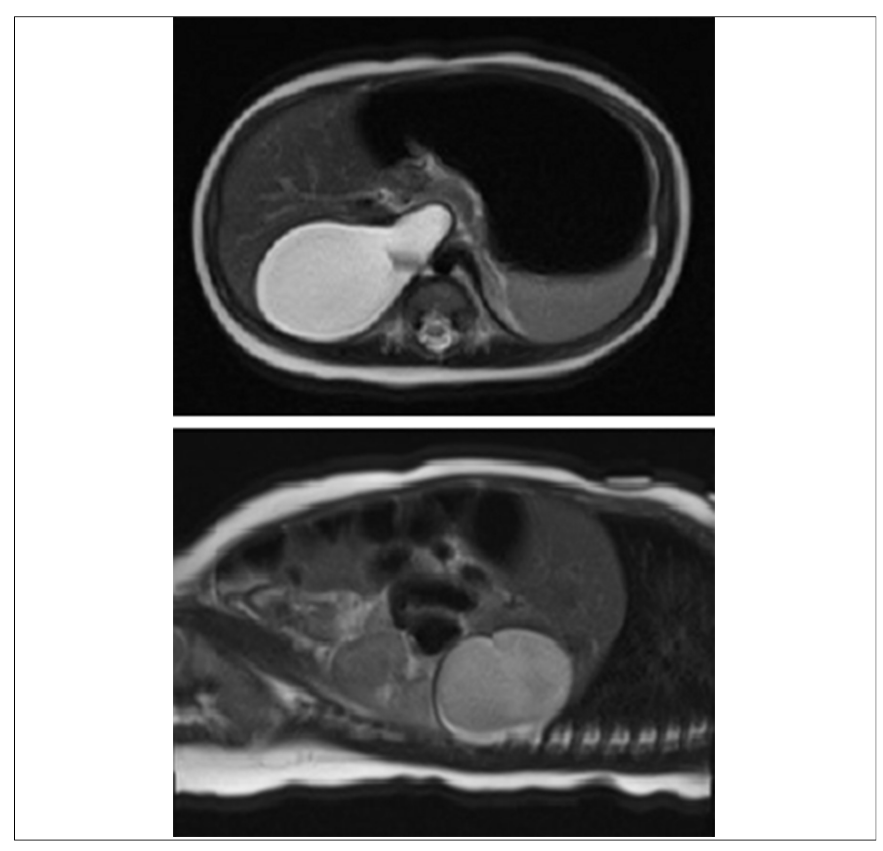

Figure 2: MRI showing heterogeneous lesion in right suprarenal region.

\section{DISCUSSION}

Enteric duplication cysts are rare congenital enteric malformations characterised by cystic or tubular appearance, a well-developed coat of smooth muscle, and an epithelial lining representing some portions of the intestinal tract mucosa.2,3 Although ileocecal region is most common site of enteric duplication, GDC makes up approximately $4 \%-9 \%$ of gastrointestinal duplications and occurs in both circular and tubular form.2-5 Most of the GDC-like enteric duplications are intra-abdominal, solitary, spherical or elliptical in shape, share common blood supply and are attached to adjacent bowel wall with separated mucosa with greater curvature being the most common site of origin. ${ }^{3}$ As experienced in present and handful of reported cases, GDC may present as isolated retroperitoneal cyst without any communication or vascular attachment to stomach. 4

Embryogenesis of GDC has been explained on the basis of different theories like segmental twinning theory, recanalisation barrier of the alimentary tract, persistence of fetal gut diverticula, and separation barrier of the notochord and archenteron. ${ }^{2-5}$ Surprisingly, development of isolated GDC in retroperitoneum remains unclear and can only be explained based on embryological theory postulated for isolated enteric duplication cyst. 4 The presence of isolated GDC in antenatal period further supports the hypothesis of presence of either heterotrophic mucosa in retroperitoneum or torsion of diverticulum in early embryogenesis as possible factor in its development. $3-5$

Histopathology in GDC is characterised by presence of gastric mucosa, smooth muscle in the wall, sharing of blood supply with stomach along with communication with gastric lumen or not.2,3 Isolated GDC neither communicates nor has any attachment with gastric wall. As
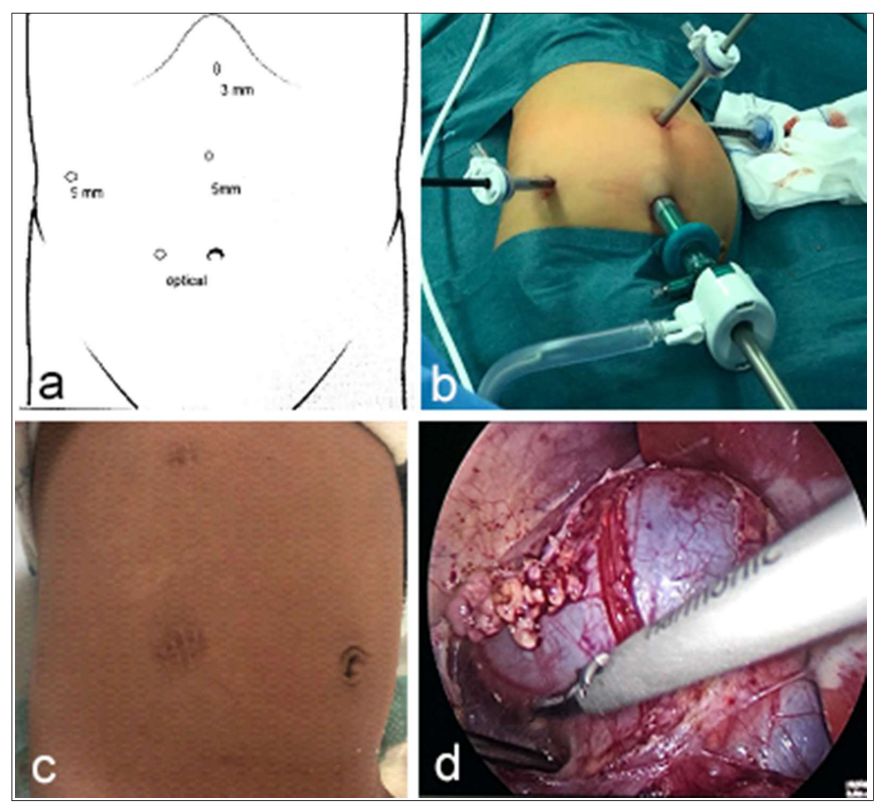

Figure 3: $(a, b, c)$ Port placement. (d) Intraoperative findings. 
experienced in the present and already reported cases, the diagnosis in such cases confirmed histologically by the presence of smooth muscle, both circular and longitudinal, along with gastrointestinal lining consistent with gastric mucosa. $1,2,3,6,7$

The clinical presentation of isolated GDC is variable. ${ }^{3}$ Most cases are diagnosed by 2 years of age presenting with features of abdominal pain, vomiting and abdominal swelling. 1-4,7 Those who remain asymptomatic present in adulthood. A review of literature and experience with the present case suggests a shift towards early age of presentation, which can be attributed to advancement in feto-maternal care, thus making it feasible to diagnose the presence of intra-abdominal mass early in pregnancy and; hence, prompting further planned evaluation in postnatal period. $1,2,4,9$ In comparison to typical GDC, a lack of communication with gastric lumen with presence of active secretory gastric epithelial lining explains early presentation and lack of features like hematemesis in cases of isolated GDC. Similarly, although associated anomalies including genitourinary and vertebral anomalies have been reported with GDC with incidence of $35-50 \%$, but as experienced in present and reported cases, isolated retroperitoneal GDC is rarely associated with other anomalies.6,8

The diagnosis of isolated GDC is confirmed on intraoperative findings and histopathological examination. $1,3,8$ It is difficult to diagnose GDC in infantile period even with advanced imaging modalities including ultrasound, CT or MRI, and it is often misdiagnosed as pancreatic cyst, renal cyst, adrenal cyst, or cystic neuroblastoma. ${ }^{1-6,8}$ Ultrasound examination remains initial modality of choice for most cases of intra-abdominal mass. Muscular rim sign remains diagnostic sign on ultrasound examination in experienced hands. 1,3,4,7 In present case, the intra- abdominal cystic mass was diagnosed as renal cyst in antenatal period and underwent further evaluation as either adrenal cyst or cystic neuroblastoma on MRI imaging. As experienced in present and reported cases, intraoperative findings provide a clue to diagnosis, which is confirmed only on histological examination of resected specimen.

The treatment of GDC is mainly surgical owing to potential of development of ulceration, intra-luminal bleeding, perforation, malignancy and most commonly progressive increase in size and mass effect due to fluid accumulation, especially in non-communicating isolated GDC.1,3,6,7 Complete surgical excision of cyst either by trans-peritoneal or laparoscopic approach remains treatment of choice. As experienced in present case, only a handful of cases have been treated successfully by laparoscopic excision in infantile and neonatal age group. 1,4,9 The surgical experience with present case highlights the need to apply concept of triangulation where port placement was planned as per the nature and location of pathology taking simultaneously into consideration the patient's physical profile. The optical port placed at McBurney point instead of umbilicus with patient in lateral position facilitated direct access to retroperitoneal mass and successful excision with minimal morbidity. Similar surgical approach has been described by Jin et al. in their series of 5 cases where optical port placed off-umbilicus helped in successful laparoscopic excision of GDC. ${ }^{1}$

Isolated GDC should be considered in the differentials of cystic intra-abdominal mass, both in antenatal and postnatal period. The diagnosis of isolated GDC can be a dilemma even with advanced imaging studies. Laparoscopy is feasible even in neonatal and infants presenting with cystic retroperitoneal mass, both in confirming the diagnosis and in surgical excision with minimal morbidity and mortality. We conclude that umbilicus should not be taken as universal site for optical port entry; and proper access point should be decided as per laparoscopic principle of triangulation, only after positioning of patient along with taking into consideration the patient's physical profile, alongwith size and location of intra-abdominal mass.

\section{CONFLICT OF INTEREST:}

Authors declared no conflict of interest.

\section{AUTHORS' CONTRIBUTION:}

VG: Writer, data collection, literature review.

UJ: Data collection, literature review, design, digital processing.

GJ: Literature review, design, concept.

DM: Literature review, design, manuscript revision.

MA: Design, supervision, analysis, critical review.

\section{REFERENCES}

1. Ren HX, Duan LQ, Wu XX, Zhao BH, Jin YY. Laparoscopic resection of gastric duplication cysts in newborns: A report of five cases. BMC Surg 2017; 17:37.

2. Taiwo OC, Faturoti OI, Taiwo AO, Adefalujo A. Gastric duplication cyst in a Nigerian infant. J Case Reports 2018; 8: 42-5.

3. Pachl M, Patel K, Bowen C, Parikh D. Retroperitoneal gastric duplication cyst: A case report and literature review. Pediatr Surg Int 2012; 28:103-5.

4. Souzaki R, leiri S, Kinoshita Y, Nishie A. Laparoscopic resection of an isolated retroperitoneal enteric duplication in an infant. J Ped Surg Case Reports 2013; 1:167-70.

5. Halder P, Mandal KC, Debnath B, Mukhopadhyay M. Gastric duplication cyst in children: A case report and literature review. SM J Pediatr Surg 2016; 2:1031.

6. Bonacci JL, Schlatter MG. Gastric duplication cyst: A unique presentation. J Pe-diatr Surg 2008; 43:1203-5.

7. Hlouschek V, Domagk D, Naehrig J, Siewert JR, Domschke W. Gastric duplication cyst: A rare endo-sonographic finding in an adult. Scand J Gastroenterol 2005; 40:1129-31.

8. Singh JP, Rajdeo H, Bhuta K, Savino JA. Gastric duplication cyst: Two case reports and review of the literature. Case Rep Surg 2013; 2013:605-59.

9. Ford WD, Guelfand M, López PJ, Furness ME. Laparoscopic excision of a gastric duplication cyst detected on antenatal ultrasound scan. J Pediatr Surg 2004; 39:e8-e10. 